\title{
Efficacy of a Deep Thermal Therapy System for Osteoarthritis of the Knee
}

\author{
Kazuhiro Harada ${ }^{1}$, Kenji Takahashi ${ }^{2}$, Futoshi Ikuta ${ }^{3}$, Yasuhiro Shindo ${ }^{4}$, \\ Kazuo Kato ${ }^{5}$, Yuya Iseki ${ }^{6}$, Yasushi Oshima ${ }^{3}$ and Tokifumi Majima ${ }^{3}$

\begin{abstract}
${ }^{1}$ Department of Orthopaedic Surgery, International University of Health and Welfare Hospital, Tochigi, Japan ${ }^{2}$ Department of Orthopaedics, Graduate School of Medical Science, Kyoto Prefectural University of Medicine, Kyoto, Japan ${ }^{3}$ Department of Orthopaedic Surgery, Nippon Medical School, Tokyo, Japan ${ }^{4}$ Faculty of Science \& Engineering, Toyo University, Saitama, Japan

${ }^{5}$ Department of Mechanical Engineering Informatics, Meiji University, Kanagawa, Japan

${ }^{6}$ Department of Industrial Systems Engineering, Mechanical System Design Course, National Institute of Technology (KOSEN), Hachinohe College, Aomori, Japan
\end{abstract}

Background: This study sought to assess the efficacy of a deep-tissue thermal therapy system with a resonant cavity applicator (DTT-RCA), which safely heats deep joint tissue for treating osteoarthritis (OA) of the knee.

Methods: Two groups of participants were recruited. The DTT-RCA group comprised 20 knees. Kellgren-Lawrence (K-L) grade was I and II in 8 knees (DTT-RCA I/II group) [mean age 73.3 years (standard deviation 11.4)], III and VI in 12 knees (DTT-RCA III/IV group) [75.4 (8.6) years]. The control group comprised 13 knees [68.2 (10.8) years]. K-L grade was I in 7 knees and II in 6 knees. This group received exercise therapy. The DTT-RCA I/II group and the control group were imaged by MRI T2 mapping at baseline and 6 months to determine the area of cartilage degeneration.

Results: Visual Analogue Scale improved only in the DTT-RCA I/II post-intervention ( $p<0.01$ ). Japanese Orthopedic Association knee rating scores (DTT-RCA I/II: p < 0.01, control group: $\mathrm{p}<0.01$ ), the Japanese Knee Osteoarthritis Measure (DTT-RCA I/II: $p<0.05$, control: $p<0.01$ ), and the Knee injury and Osteoarthritis Outcome Score (DTT-RCA I/II: p < 0.01, DTT-RCA III/IV: p < 0.05, control: p < 0.01) post-intervention. The magnitude of change did not differ significantly between the three groups. The area of cartilage degeneration did not change significantly post-intervention in the DTT-RCA I/II group, not even relative to the control group.

Conclusions: This was the first study to test a DTT-RCA system in patients with knee OA. The system reduced the clinical symptoms of knee OA and could potentially be effective for conservative therapy.

(J Nippon Med Sch 2021; 88: 335-341)

Key words: knee, osteoarthritis, cartilage, conservative treatment, induced hyperthermia

\section{Introduction}

Osteoarthritis (OA) of the knee is a degenerative disease that causes pain and functional impairment. It has become a global problem because it not only causes pain and distress for patients themselves ${ }^{1}$, but also imposes a high-cost burden on healthcare systems ${ }^{2}$. No radical therapy for OA of the knee currently exists ${ }^{3}$, and it must be treated with a combination of modalities such as physical therapy, exercise therapy, orthotics, and pharmacother- apy.

Hyperthermia is widely used in routine physical therapy for musculoskeletal disorders ${ }^{4}$, but there is little high-quality evidence regarding hyperthermia for OA of the knee $^{5}$, and absolutely no clinical evidence regarding the disease-modifying effect of thermal therapy. However, recent animal studies have shown that heating of articular cartilage attenuates cartilage degeneration ${ }^{6,7}$. It is easy to adequately heat the tiny joints of small animals

Correspondence to Kenji Takahashi, MD, PhD, Department of Orthopaedics, Kyoto Prefectural University of Medicine, 465

Kawaramachi-Hirokoji, Kajii-cho, Kamigyo-ku, Kyoto, Kyoto 602-8566, Japan

E-mail: t-keji@mbox.kyoto-inet.or.jp

https://doi.org/10.1272/jnms.JNMS.2021_88-505

Journal Website (https://www.nms.ac.jp/sh/jnms/) 
Table 1 Demographic characteristics of the participants

\begin{tabular}{lccc}
\hline & DTT-RCA I/II & DTT-RCA III/IV & Control \\
\hline Sex [M/F] & $3 / 5$ & $6 / 6$ & $3 / 10$ \\
Age [years] & $73.3(11.4)$ & $75.4(8.6)$ & $68.2(10.8)$ \\
Height [cm] & $161.7(7.4)$ & $160.0(6.4)$ & $157.1(7.6)$ \\
Weight [kg] & $64.1(11.0)$ & $65.4(9.1)$ & $60.1(10.2)$ \\
BMI [kg/m²] & $24.4(2.7)$ & $25.5(3.0)$ & $24.2(2.3)$ \\
\hline
\end{tabular}

Mean (standard deviation). Age, Height, Weight, BMI: No significant difference in any parameter (Kruskal-Wallis test). DTT-RCA: Deep-tissue heating therapy using resonant cavity applicator

such as rats and guinea pigs, but difficult to heat cartilage in larger human knee joints. Superficial thermal therapy (e.g., hot baths, hot packs, and paraffin wax baths) heats only the skin and subcutaneous tissue, and do not reach the deep tissue of the joints. ${ }^{8}$. Microwave diathermy systems do not easily heat deep joint tissue either because most of the electromagnetic energy is absorbed at the surface of the affected area'. Large skincontact bipolar applicators that wrap around the entire knee can heat the deep tissue of the $k_{n e} e^{10}$, but are not practical for routine use due to their large size and high cost. There are also safety concerns about electromagnetic waves escaping outside the targeted area. Issues with modalities may be a large reason why few studies to date have reported the effectiveness of thermal therapy for $\mathrm{OA}$ of the knee.

A deep-tissue thermal therapy system using a resonant cavity applicator (DTT-RCA) is in development to address this problem by establishing a thermal therapy modality that can safely heat deep joint tissue. A study that analyzed the distribution of temperature in the body using a finite element method model numerically confirmed the potential to achieve deeper heat penetration than that possible with existing microwave therapy devices used in the clinic ${ }^{11}$. This potential has also been demonstrated experimentally by testing a prototype system on muscle phantoms made of agar ${ }^{12-14}$. Other reports have confirmed the performance of a DTT-RCA system using a phantom and then validated its safety and efficacy in knees of healthy volunteers ${ }^{11,15,16}$. However, no study has ever tested DTT-RCA in patients with OA of the knee, and thus its effects on OA symptoms and articular cartilage are unknown.

The aim of this study was to assess the efficacy of 6 months of treatment with DTT-RCA for OA of the knee. The hypothesis was that joint pain would decrease and function would improve post-intervention.

\section{Materials and Methods}

The study protocol was approved by the ethics committees of our institutions and the study was conducted in accordance with the Declaration of Helsinki (Approval Number: 13-B-248). The participants were informed that data from the study would be submitted for publication, and all gave their consent.

Participants were patients who visited the hospital for knee pain and received a diagnosis of OA of the knee. Twenty knees of 20 patients (9 men, 11 women) with a mean age of 74.6 years (standard deviation 9.6) and mean body mass index (BMI) of 25.1 (2.9) were assigned to the DTT-RCA group, and received DTT-RCA for 6 months. The Kellgren-Lawrence (K-L) grade, which indicates the stage of knee OA was I in 5 knees and II in 3 knees (DTT-RCA I/II group), III in 6 knees and VI in 6 knees (DTT-RCA III/IV group). Thirteen knees of 13 patients (3 men, 10 women) with a mean age of 68.2 (10.8) years and mean BMI of 24.2 (2.3) were assigned to the control group and received exercise therapy for 6 months. The K-L grade in this group was I in 7 knees and II in 6 knees. Age, height, weight, and BMI did not differ significantly between the three groups (Table 1). Individuals with a history of severe trauma or surgery involving the lower extremities, individuals who were currently receiving treatment, and individuals who did not give informed consent were excluded.

At 6 months post-intervention, questionnaires were administered, and knee cartilage degeneration was assessed qualitatively using MRI T2 mapping. However, assessment of degeneration with MRI T2 mapping was not feasible in patients with a K-L grade of III or higher due to marked loss of cartilage. Therefore, only the DTT-RCA I/ II group were evaluated for degeneration.

The 5 questionnaires administered were the Visual Analogue Scale (VAS), the Japanese Orthopedic Association knee rating score (JOA score), the Knee injury and Osteoarthritis Outcome Score (KOOS), the Japanese Knee 


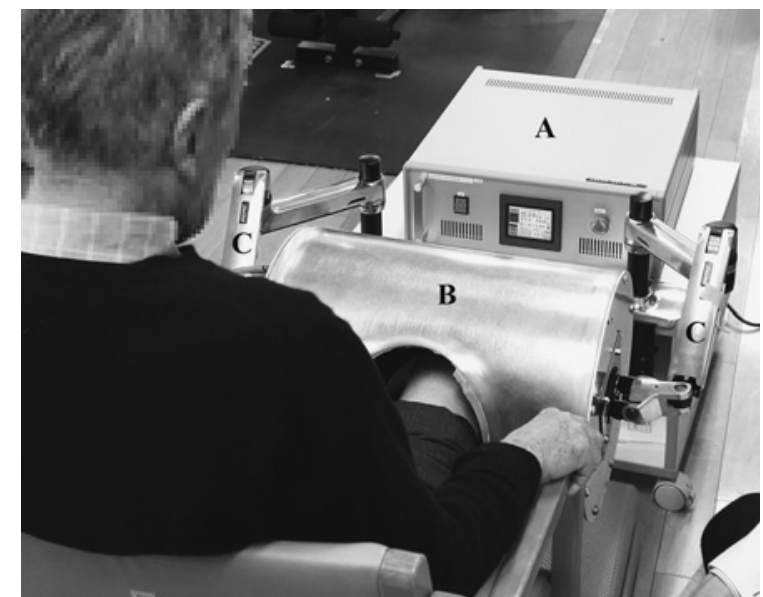

Fig. 1 Setup of deep thermal system using resonant cavity applicator

A: High-frequency amplifier (including an impedance matching unit), B: Resonant cavity applicator, C: adjustable arms

Osteoarthritis Measure (JKOM), and the Tegner activity scale (Tegner).

MR examinations were performed using a 3.0-T MR unit (Achieva 3.0T; Philips Medical Systems, Best, Netherlands). Patients were placed with the knee in about $15^{\circ}$ flexion and coronal images were acquired for slices parallel to a line connecting the edges of the medial and lateral posterior tibial condyles. Multi-echo spin echo images (repetition time/echo time, 4,613 ms/ $10 \times \mathrm{n}(\mathrm{n}=1-8)$ slice thickness $3 \mathrm{~mm}$; field of view $14 \mathrm{~cm}$; matrix $256 \times$ 256) were acquired for T2-mapping. To avoid the partial volume effect of fluid collection near the articular cartilage, voxels showing $\mathrm{T} 2$ values $>130 \mathrm{~ms}$ were excluded. On a coronal T2 mapping image, the slice showing the medial meniscal body in the smallest size was selected as the central slice. This slice and 2 more adjacent slices at $5 \mathrm{~mm}$ anterior and $5 \mathrm{~mm}$ posterior to the central slice were used for cartilage assessment. From each slice, the medial femoral cartilage and medical tibial cartilage were extracted by direct segmentation using proton densityweighted images as reference. For each of the extracted cartilages, parts with a T2 value between $50 \mathrm{~ms}$ and 130 ms were considered to represent degenerative change and the area was calculated using workstation software (Aze ${ }^{\circledR}$; VirtualPlace AZE, Ltd., Tokyo, Japan). The total area of cartilage degeneration was calculated as the sum of areas of the 3 parts calculated with this method. To standardize the procedure, all measurements were performed by a single investigator.

\section{DTT-RCA Group}

Patients in the DTT-RCA group were treated with DTT-
RCA once every 2 weeks. The knee was heated for 20 $\min$ at a power of 20-40 W. The resonance frequency fluctuates in accordance with the size of the patient's knee and the position of the device, but the auto-tuning system of the prototype device automatically tunes the resonance frequency to within the set frequency range, enabling stable heating. The frequency range was set to 420-470 MHz (Fig. 1) ${ }^{17}$.

\section{Control Group}

The control group was assigned to do 5 exercises: 1) ankle range of motion exercise involving active plantarand dorsiflexion of the ankle while sitting with the legs stretched out on the ground; 2) knee range of motion exercise involving passive flexion and extension of the knee while sitting with the legs stretched out on the ground; 3) quadriceps strengthening exercise involving extension of the knee while sitting with legs stretched out on the ground or on a bed; 4) hip abduction exercise involving abduction of the hip in a lateral recumbent position; and 5) squats. A physical therapist guided patients through these exercises and checked their form once a week. Patients performed 3 sets of 10 repetitions of these exercises daily.

\section{Statistical Analysis}

Due to the small sample size, nonparametric tests were selected for all analyses. The Wilcoxon signed-rank test was used to compare pre- and post-intervention values and the Kruskal-Wallis test was used to compare the magnitude of change between the three groups. MannWhitney $U$ test was applied to the change of cartilage degeneration area to compare between the DTT-RCA I/II and control groups. Significance was set at $\alpha=0.05$ for all tests. We used SPSS Statistical Package (Version 23; SPSS, Chicago, IL) for all statistical analysis.

\section{Results}

The magnitude of change in VAS were -16.7 [95\% confidence interval: $-26.9,-6.4$ ] in the DTT-RCA I/II group (p $<0.01), 6.3[-8.5,21.0]$ in the DTT-RCA III/IV, and -10.8 $[-28.2,6.5]$ in the control group (same order of results below). The magnitude of change in JOA score were 10.6 $[6.7,14.5](\mathrm{p}<0.01) / 5.0[-4.7,14.7] / 8.5[3.6,13.3](\mathrm{p}<$ $0.01)$, JKOM were $-16.8[-25.0,-8.5](\mathrm{p}<0.05) /-4.2$ $[-13.1,4.8] /-14.5[-20.3,-8.8](\mathrm{p}<0.01)$, KOOS were $15.0[8.3,21.6](\mathrm{p}<0.01) / 6.7[0.8,12.6](\mathrm{p}<0.05) / 14.7$ $[9.6,19.9](\mathrm{p}<0.01)$, and Tegner were $0.3[-0.5,1.0] /-0.3$ $[-0.9,0.2] /-0.2[-0.9,0.5]$. The magnitude of change did not differ significantly between the three groups (Table 2). 
Table 2 Pre- and post-intervention questionnaire results for Deep Thermal System (DTT-RCA) group

\begin{tabular}{clccc}
\hline Score & & Pre-intervention & Post-intervention & (Post) - (Pre) \\
\hline \multirow{2}{*}{ VAS } & DTT-RCA I/II** & $39.1[28.1,50.1]$ & $22.4[12.5,32.3]$ & $-16.7[-26.9,-6.4]$ \\
& DTT-RCA III/IV & $37.3[27.4,47.1]$ & $43.5[32.9,54.1]$ & $6.3[-8.5,21.0]$ \\
& Control & $51.2[40.6,61.7]$ & $40.3[24.4,56.2]$ & $-10.8[-28.2,6.5]$ \\
\multirow{2}{*}{ JOA } & DTT-RCA I/II** & $83.8[78.6,88.9]$ & $94.4[90.5,98.3]$ & $10.6[6.7,14.5]$ \\
& DTT-RCA III/IV & $76.7[68.3,85.1]$ & $81.7[75.5,87.9]$ & $5.0[-4.7,14.7]$ \\
& Control** & $79.2[74.2,84.3]$ & $87.7[83.5,91.9]$ & $8.5[3.6,13.3]$ \\
\multirow{3}{*}{ KOM } & DTT-RCA I/II* & $25.9[18.3,33.4]$ & $9.1[6.4,11.9]$ & $-16.8[-25.0,-8.5]$ \\
& DTT-RCA III/IV & $39.1[30.8,47.3]$ & $34.9[22.4,47.5]$ & $-4.2[-13.1,4.8]$ \\
& Control** & $32.2[24.0,40.3]$ & $17.6[14.4,20.9]$ & $-14.5[-20.3,-8.8]$ \\
& DTT-RCA I/II** & $68.7[60.2,77.1]$ & $83.6[78.1,89.2]$ & $15.0[8.3,21.6]$ \\
& DTT-RCA II/IV* & $56.3[49.3,63.2]$ & $63.0[54.3,71.7]$ & $6.7[0.8,12.6]$ \\
\multirow{2}{*}{ Tegner } & Control** & $58.2[50.7,65.7]$ & $72.9[67.5,78.4]$ & $14.7[9.6,19.9]$ \\
& DTT-RCA I/II & $2.1[1.4,2.8]$ & $2.4[2.0,2.7]$ & $0.3[-0.5,1.0]$ \\
& DTT-RCA III/IV & $1.9[1.5,2.4]$ & $1.6[1.2,2.0]$ & $-0.3[-0.9,0.2]$ \\
& Control & $2.7[1.9,3.5]$ & $2.5[1.9,3.2]$ & $-0.2[-0.9,0.5]$ \\
\hline
\end{tabular}

Means value [95\% confidence interval]. Significant improvement after intervention: ${ }^{*} \mathrm{p}<0.05$, ${ }^{* *} \mathrm{p}<0.01$. The magnitude of change did not differ significantly between the three groups. Lower scores are better for VAS and JKOM, and higher scores are better for JOA, KOOS, and Tegner. A negative magnitude of change indicates improvement for the first 2, and a positive magnitude of change for the last 3. VAS, Visual Analogue Scale; JOA, The Japanese Orthopedic Association knee rating score; JKOM, Japanese Knee Osteoarthritis Measure; KOOS, Knee injury and Osteoarthritis Outcome Score; Tegner, Tegner activity scale.

Table 3 Area of cartilage degeneration determined by MRI T2 mapping $\left(\mathrm{mm}^{2}\right)$

\begin{tabular}{lccc}
\hline & Pre-intervention & Post-intervention & (Post) - (Pre) \\
\hline DTT-RCA I/II & $184.3[152.9,215.8]$ & $179.9[160.0,199.7]$ & $-4.5[-25.5,16.6]$ \\
Control & $145.3[125.6,164.9]$ & $160.4[135.8,185.0]$ & $15.1[0.1,30.2]$ \\
ALL & $160.2[141.6,178.7]$ & $167.8[150.7,185.0]$ & $7.7[-5.0,20.3]$ \\
\hline
\end{tabular}

Mean value [ $95 \%$ confidence interval]. There was no significant difference postintervention and no significant difference in the magnitude of change between groups. DTT-RCA I/II: Kellgren-Lawrence grade I and II patients treated with deep-tissue thermal therapy using a resonant cavity applicator.

The area of cartilage degeneration on MRI T2 mapping in the DTT-RCA I/II group was $184.3 \mathrm{~mm}^{2}$ [152.9, 215.8] pre-intervention and $179.9 \mathrm{~mm}^{2}$ [160.0, 199.7] postintervention, and the magnitude of change was $-4.5 \mathrm{~mm}^{2}$ $[-25.5,16.6]$. In the control group, results (same order) were $145.3 \mathrm{~mm}^{2}$ [125.6, 164.9], $160.4 \mathrm{~mm}^{2}$ [135.8, 185.0], and $15.1 \mathrm{~mm}^{2}[0.1,30.2]$. The mean area of degeneration decreased in the DTT-RCA I/II group post-intervention, but not significantly so, and the magnitude of change did not differ significantly between groups, either (Table 3, Fig. 2). No patient complained of adverse events due to DTT-RCA.

\section{Discussion}

There is little evidence for thermal therapy in treating
OA of the knee. The latest Osteoarthritis Research Society International guidelines rate the quality of evidence as "very low" ${ }^{5}$. However, thermal therapy has been shown to reduce pain and improve function in various studies of treatments such as superficial local hot and cold application $^{18}$, local microwave diathermy ${ }^{19}$, and spa therapy ${ }^{20}$. In this study, we found that thermal therapy did not reduce knee pain but did improve function and reduce symptoms. OA of the knee is caused by not only factors inside the knee joint, but also factors in extra-articular soft tissue $\mathrm{e}^{21}$ and periarticular tissue $\mathrm{e}^{22,23}$. A temperature increase of $1^{\circ} \mathrm{C}$ increases metabolic activity; an increase of $2^{\circ} \mathrm{C}-3^{\circ} \mathrm{C}$ reduces muscle spasms, increases blood flow, and reduces chronic inflammation; an increase of $4^{\circ} \mathrm{C}$ enhances the viscoelastic properties of collagen ${ }^{24}$. This may 


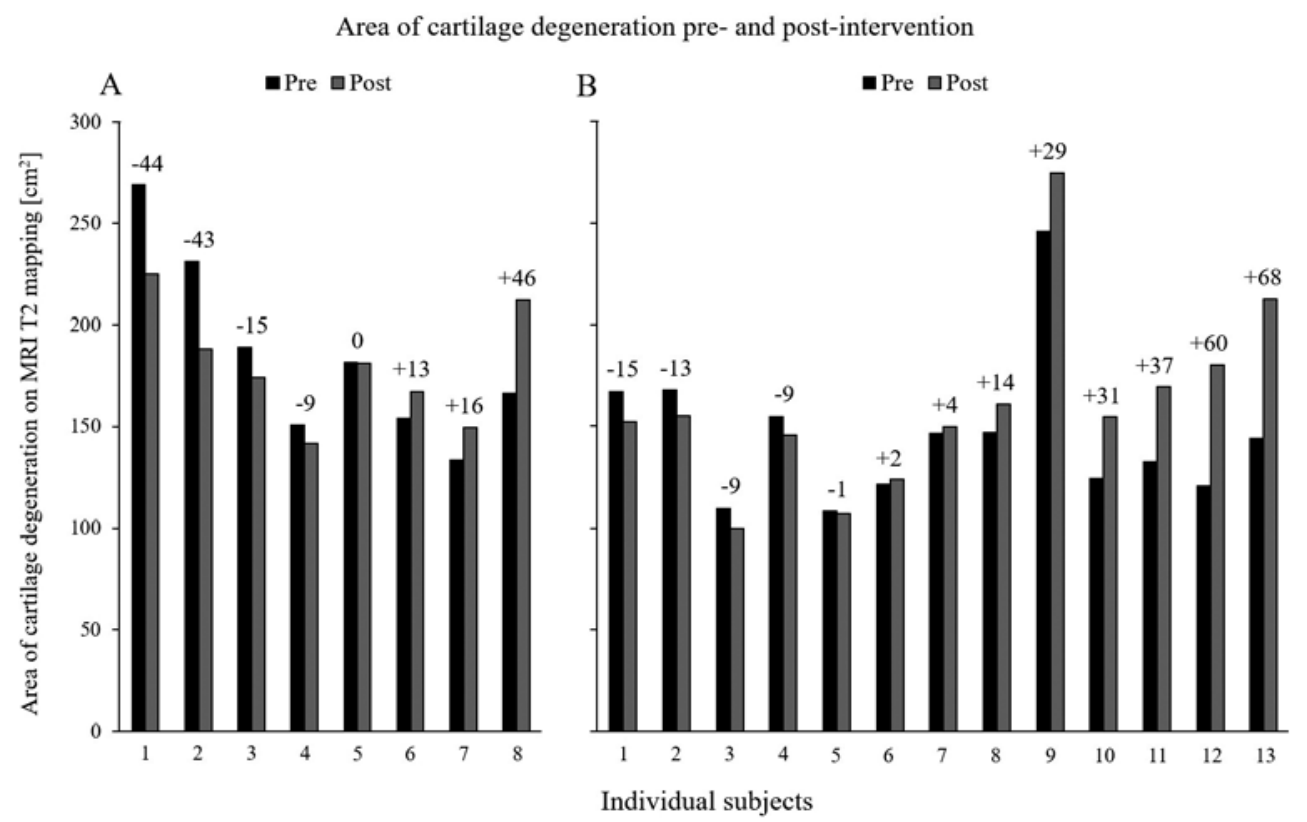

Fig. 2 A: Deep-tissue thermal therapy system with a resonant cavity applicator (DTT-RCA I/II) group, B: Control group. Area of cartilage degeneration decreased in 2 patients in the DTT RCA I/II group (A1 and A2).

be why superficial thermal therapy reduced periarticular pain, but deep-tissue thermal therapy with the DTT-RCA system did not. This result seems logical considering that articular cartilage does not contain nerves or blood vessels. However, studies in animal models have shown the following: deep-tissue thermal therapy improves matrix metabolism in articular cartilage; heat shock protein 70 produced by chondrocytes in response to thermal stimulation protects cartilage by slowing apoptosis of chondrocytes, which is a critical element in progression of OA: and deep-tissue thermal therapy strongly upregulates expression of proteoglycans and type II collagen, which are key components of the cartilage matrix ${ }^{6,25-28}$. This indicates that the improvement in function and reduction of symptoms we observed in this study may have been produced through a different mechanism than superficial thermal therapy.

The area of cartilage degeneration did not change significantly post-intervention in the DTT-RCA I/II group, not even relative to the control group. One study found that application of moist heat by heat- and steamgenerating sheets for $6 \mathrm{~h}$ a day for 12 weeks significantly reduced MRI T2 values ${ }^{29}$. The results of that study showed the potential positive effects of thermal therapy on cartilage metabolism. In the present study, we performed deep-tissue thermal therapy only once every 2 weeks in 20-min sessions. In another study, we found that $20 \mathrm{~min}$ of DTT-RCA produced a temperature in- crease of about $5^{\circ} \mathrm{C}$ in an agar phantom, and a comparable increase of about $5^{\circ} \mathrm{C}$ in healthy volunteers as evaluated by image displacement (unpublished data). In the present study, we emphasized safety over efficacy because, to our knowledge, it was the first study to be done in patients with $\mathrm{OA}$ of the knee. However, it may be acceptable to increase the duration or frequency of DTT-RCA because the protocol treatment did not cause any adverse events. One noteworthy finding is that 2 patients in the DTT-RCA I/II group but no patients in the control group had the cartilage degeneration area decrease by at least $40 \mathrm{~mm}^{2}$ (Fig. 2). This could indicate that heating by DTT-RCA affected proteoglycan and type II collagen expression because MRI T2 mapping findings correlate with fluid content in cartilage and with type II collagen $^{30}$. Exercise therapy is an essential component of conservative therapy for OA of the knee ${ }^{5}$ but is unlikely to directly impact degenerated cartilage. Future studies should analyze the factors that reduce cartilage degeneration in deep-tissue thermal therapy and the characteristics of patients who benefit from the therapy.

Several limitations of our study need to be considered. First is the insufficient sample size. The effect size for the magnitude of change in MRI T2 values in the DTT-RCA I/II group was 0.267 , and statistical power was 0.101 . Assessment of 112 knees would thus have been necessary to achieve a power of 0.8 to demonstrate a significant difference in the area of degeneration post-intervention 
when $\alpha=0.05$. Second is that we did not consider factors that affect cartilage degeneration. Due once again to the insufficient sample size, we were unable to adjust for factors such as BMI, exercise habits, level of activity at work, and disease duration in analysis of treatment effect. Third is that we only assessed cartilage degeneration based on MRI T2 values in patients with early-stage OA of the knee. This was unavoidable because assessment using T2 values is not feasible in late-stage disease. Consequently, we do not know the effect of DTT-RCA on highly degenerated articular cartilage. Nonetheless, the main strength of our study is that the heating mechanism and efficacy of the DTT-RCA system has been established in prior studies. Up to this point, thermal therapy has generally been considered ineffective ${ }^{5}$. This study has the potential to reverse opinions on thermal therapy if these results are used to improve the utility of the DTT-RCA system.

To our knowledge, this is the first study to test a DTTRCA system in patients with OA of the knee. With 6 months of treatment once every 2 weeks, this system improved function and reduced symptoms in patients with OA of the knee, and it was improved in early-stage OA rather than the severe-stage OA. The reason may be that cartilage still remains in early-stage OA of the knee.

In addition, the area of articular cartilage degeneration decreased in some patients with early-stage disease. These results indicate that DTT-RCA could be an effective conservative therapy for OA of the knee.

Acknowledgements: This work was supported by JSPS Grant-in-Aid for Scientific Research (C) Grant Number JP17K 01475 .

\section{Conflict of Interest: None.}

\section{References}

1. Carmona-Teres V, Moix-Queralto J, Pujol-Ribera E, et al. Understanding knee osteoarthritis from the patients' perspective: a qualitative study. BMC Musculoskelet Disord [Internet]. 2017 May 30;18(1):225. Available from: https:// www.ncbi.nlm.nih.gov/pubmed / 28558738

2. Bitton R. The economic burden of osteoarthritis. Am J Manag Care [Internet]. 2009 Sep;15(8 Suppl):S230-5. Available from: https://www.ncbi.nlm.nih.gov/pubmed/ 19817509

3. Roos EM, Arden NK. Strategies for the prevention of knee osteoarthritis. Nat Rev Rheumatol [Internet]. 2016 Feb;12(2):92-101. Available from: https:/ /www.ncbi.nlm.n ih.gov/pubmed/26439406

4. Sarzi-Puttini P, Cimmino MA, Scarpa R, et al. Osteoarthritis: an overview of the disease and its treatment strategies. Semin Arthritis Rheum [Internet]. 2005 Aug;35(1
Suppl 1):1-10. Available from: https://www.ncbi.nlm.nih. gov/pubmed/16084227

5. Bannuru RR, Osani MC, Vaysbrot EE, et al. OARSI guidelines for the non-surgical management of knee, hip, and polyarticular osteoarthritis. Osteoarthritis Cartilage [Internet]. 2019 Nov;27(11):1578-89. Available from: https://w ww.ncbi.nlm.nih.gov/pubmed/31278997

6. Fujita S, Arai $Y$, Nakagawa $S$, et al. Combined microwave irradiation and intraarticular glutamine administrationinduced HSP70 expression therapy prevents cartilage degradation in a rat osteoarthritis model. J Orthop Res [Internet]. 2012 Mar;30(3):401-7. Available from: https:// www.ncbi.nlm.nih.gov/pubmed /21853458

7. Takahashi K, Nakamura H, Ozawa H, et al. Effectiveness of radiofrequency hyperthermia for treating cartilage in guinea pigs with primary osteoarthritis. Cartilage [Internet]. 2018 Jan;9(1):71-9. Available from: https://www.ncb i.nlm.nih.gov/pubmed/29219022

8. Draper DO, Hopkins TJ. Increased intramuscular and intracapsular temperature via ThermaCare Knee Wrap application. Med Sci Monit [Internet]. 2008 Jun;14(6):PI7-11. Available from: https://www.ncbi.nlm.nih.gov/pubmed/ 18509284

9. Heerink WJ, Solouki AM, Vliegenthart R, et al. The relationship between applied energy and ablation zone volume in patients with hepatocellular carcinoma and colorectal liver metastasis. Eur Radiol [Internet]. 2018 Aug;28 (8):3228-36. Available from: https://www.ncbi.nlm.nih.go v/pubmed/29536242

10. Takahashi K, Kurosaki H, Hashimoto S, Takenouchi K, Kamada T, Nakamura $\mathrm{H}$. The effects of radiofrequency hyperthermia on pain and function in patients with knee osteoarthritis: a preliminary report. J Orthop Sci [Internet]. 2011 Jul;16(4):376-81. Available from: https://www.n cbi.nlm.nih.gov/pubmed/21611800

11. Shindo Y, Kato K, Ichishima Y, et al. Evaluation of deep thermal rehabilitation system using resonant cavity applicator during knee experiments. Conf Proc IEEE Eng Med Biol Soc [Internet]. 2018 Jul;2018:3220-3. Available from: h ttps://www.ncbi.nlm.nih.gov/pubmed/30441077

12. Yokoyama K, Kato K, Igarashi W, et al. Heating properties of a new hyperthermia system for deep tumors without contact. Conf Proc IEEE Eng Med Biol Soc [Internet]. 2011;2011:310-3. Available from: https://www.ncbi.nlm.ni h.gov/pubmed/22254311

13. Shindo Y, Kato K, Hirashima T, Yabuhara T. Development of automatic impedance matching system for hyperthermia treatment using resonant cavity applicator. Conf Proc IEEE Eng Med Biol Soc [Internet]. 2008;2008:4376-9. Available from: https://www.ncbi.nlm.nih.gov/pubmed/ 19163683

14. Shindo Y, Kato K, Tsuchiya K, et al. Heating properties of re-entrant resonant applicator for brain tumor by electromagnetic heating modes. Conf Proc IEEE Eng Med Biol Soc [Internet]. 2007;2007:3609-12. Available from: https:// www.ncbi.nlm.nih.gov/pubmed/18002778

15. Shindo Y, Kato K, Ikuta F, Takahashi K. Validation of noninvasive ultrasound temperature measurement system through experiments using resonant cavity applicator. Conf Proc IEEE Eng Med Biol Soc [Internet]. 2019 Jul; 2019:2521-4. Available from: https://www.ncbi.nlm.nih.g ov/pubmed/31946410

16. Shindo Y, Iseki Y, Yokoyama K, et al. SAR analysis of the improved resonant cavity applicator with electrical shield and water bolus for deep tumors by a 3-D FEM. Conf Proc IEEE Eng Med Biol Soc [Internet]. 2012;2012:5679-82. 
Available from: https://www.ncbi.nlm.nih.gov/pubmed/ 23367219

17. Shindo $Y$, Kato $K$, Tsuchiya $K$, Hirashima T, Suzuki M. Improvement of the matching speed of AIMS for development of an automatic totally tuning system for hyperthermia treatment using a resonant cavity applicator. Conf Proc IEEE Eng Med Biol Soc [Internet]. 2009;2009: 3072-5. Available from: https://www.ncbi.nlm.nih.gov/p ubmed/19963559

18. Aciksoz S, Akyuz A, Tunay S. The effect of selfadministered superficial local hot and cold application methods on pain, functional status and quality of life in primary knee osteoarthritis patients. J Clin Nurs [Internet]. 2017 Dec;26(23-24):5179-90. Available from: https:// www.ncbi.nlm.nih.gov/pubmed/28880416

19. Rabini A, Piazzini DB, Tancredi G, et al. Deep heating therapy via microwave diathermy relieves pain and improves physical function in patients with knee osteoarthritis: a double-blind randomized clinical trial. Eur J Phys Rehabil Med [Internet]. 2012 Dec;48(4):549-59. Available from: https://www.ncbi.nlm.nih.gov/pubmed/22820 824

20. Forestier R, Erol Forestier FB, Francon A. Spa therapy and knee osteoarthritis: A systematic review. Ann Phys Rehabil Med [Internet]. 2016 Jun;59(3):216-26. Available from: https://www.ncbi.nlm.nih.gov/pubmed/26996955

21. Dye SF, Vaupel GL, Dye CC. Conscious neurosensory mapping of the internal structures of the human knee without intraarticular anesthesia. Am J Sports Med [Internet]. 1998 Nov-Dec;26(6):773-7. Available from: https://w ww.ncbi.nlm.nih.gov/pubmed/9850777

22. Toktas H, Dundar U, Adar S, Solak O, Ulasli AM. Ultrasonographic assessment of pes anserinus tendon and pes anserinus tendinitis bursitis syndrome in patients with knee osteoarthritis. Mod Rheumatol [Internet]. 2015 Jan; 25(1):128-33. Available from: https://www.ncbi.nlm.nih.g ov/pubmed / 25036227

23. Tran J, Peng PWH, Lam K, Baig E, Agur AMR, Gofeld M. Anatomical study of the innervation of anterior knee joint capsule: Implication for image-guided intervention. Reg Anesth Pain Med [Internet]. 2018 May;43(4):407-14. Available from: https://www.ncbi.nlm.nih.gov/pubmed/29557 887

24. Draper DO. Ten mistakes commonly made with ultrasound use: Current research sheds light on myths. Athletic Training. 1996 [Internet]. 1996;2:95-107. Available from: https://ci.nii.ac.jp/naid/10007758010/

25. Arai $Y$, Kubo T, Kobayashi K, et al. Adenovirus vectormediated gene transduction to chondrocytes: in vitro evaluation of therapeutic efficacy of transforming growth factor-beta 1 and heat shock protein 70 gene transduction. J Rheumatol [Internet]. 1997 Sep;24(9):1787-95. Available from: https:/ / www.ncbi.nlm.nih.gov/pubmed/9292805

26. Kubo T, Arai Y, Takahashi K, et al. Expression of transduced HSP70 gene protects chondrocytes from stress. J Rheumatol [Internet]. 2001 Feb;28(2):330-5. Available from: https://www.ncbi.nlm.nih.gov/pubmed/11246671

27. Terauchi R, Takahashi KA, Arai Y, et al. Hsp70 prevents nitric oxide-induced apoptosis in articular chondrocytes. Arthritis Rheum [Internet]. 2003 Jun;48(6):1562-8. Available from: https://www.ncbi.nlm.nih.gov/pubmed/12794 824

28. Tonomura $\mathrm{H}$, Takahashi KA, Mazda $\mathrm{O}$, et al. Glutamine protects articular chondrocytes from heat stress and NOinduced apoptosis with HSP70 expression. Osteoarthritis Cartilage [Internet]. 2006 Jun;14(6):545-53. Available from: https://www.ncbi.nlm.nih.gov/pubmed/16480901

29. Ochiai S, Watanabe A, Oda H, Ikeda H. Effectiveness of thermotherapy using a heat and steam generating sheet for cartilage in knee osteoarthritis. J Phys Ther Sci [Internet]. 2014 Feb;26(2):281-4. Available from: https://www.n cbi.nlm.nih.gov/pubmed/24648649

30. Verstraete KL, Almqvist F, Verdonk $\mathrm{P}$, et al. Magnetic resonance imaging of cartilage and cartilage repair. Clin Radiol [Internet]. 2004 Aug;59(8):674-89. Available from: h ttps://www.ncbi.nlm.nih.gov/pubmed/15262541

(Received, May 1, 2020)

(Accepted, September 11, 2020)

(J-STAGE Advance Publication, September 30, 2020)

Journal of Nippon Medical School has adopted the Creative Commons Attribution-NonCommercial-NoDerivatives 4.0 International License (https://creativecommons.org/licenses/by-nc-nd/4.0/) for this article. The Medical Association of Nippon Medical School remains the copyright holder of all articles. Anyone may download, reuse, copy, reprint, or distribute articles for non-profit purposes under this license, on condition that the authors of the articles are properly credited. 